\title{
Shower reconstruction with the KM3NeT/ORCA detector
}

\author{
Jannik Hofestädt* \\ for the KM3NeT Collaboration \\ Friedrich-Alexander University of Erlangen-Nürnberg, \\ Erlangen Centre for Astroparticle Physics \\ E-mail: Jannik.Hofestaedtephysik.uni-erlangen.de
}

\begin{abstract}
The neutrino mass hierarchy can be experimentally determined by measuring the zenith angle and energy dependent oscillation pattern of $\mathrm{GeV}$-scale atmospheric neutrinos that have traversed the Earth. ORCA (Oscillation Research with Cosmics in the Abyss) is a planned multi-megaton underwater Cherenkov detector in the Mediterranean Sea that will use the technology developed for $\mathrm{KM} 3 \mathrm{NeT}$ to perform this measurement.

The reconstruction of neutrino induced shower-like events, in particular from electron neutrinos in charged-current interactions, is a key task and substantially affects the mass hierarchy sensitivity. In this paper the methodology and performance of a shower reconstruction developed for ORCA is discussed. Shower energy, direction and the interaction inelasticity are reconstructed via a maximum likelihood fit. It is shown that the properties of the deep sea water allow for good direction and energy resolutions with large effective volume.

The presented study also indicates that one can gain access to the inelasticity in shower-like events. This allows to exploit the different light yields in electromagnetic and hadronic showers improving the energy resolution and it may allow a separation of neutrino and anti-neutrino charged-current and of neutral-current events on a statistical basis, and consequently enhances the sensitivity to the neutrino mass hierarchy.
\end{abstract}

The 34th International Cosmic Ray Conference,

30 July- 6 August, 2015

The Hague, The Netherlands

${ }^{*}$ Speaker. 


\section{Introduction}

Since the measurement of the neutrino mixing angle $\theta_{13}$, the determination of the neutrino mass hierarchy $(\mathrm{NMH})$, i.e. the ordering of the mass eigenstates, has become a central goal of upcoming neutrino oscillation experiments. Recently, it has been suggested that underwater (ice) Cherenkov detectors with a relatively modest volume might be able to provide significant sensitivity to the NMH by using the flux of atmospheric neutrinos that have passed through the Earth towards the detector [1]. Due to matter-induced oscillation effects in the Earth in conjunction with different cross-sections and atmospheric neutrino fluxes for neutrinos and antineutrinos, the expected event rates of neutrinos in the energy regime of about $3-20 \mathrm{GeV}$ is different for normal and inverted hierarchy. Therefore, depending on the experimental resolutions of neutrino energy and zenith angle - the latter defining the neutrino path length through the Earth - a determination of the NMH is feasible.

Additional sensitivity to the NMH is possible if the interaction inelasticity $y$ can be measured, as $v$ and $\bar{v}$ interactions show different inelasticity distributions. This is discussed for ${ }^{c} \vec{v}_{\mu}$ in [2].

Two distinct signatures in the detector can be considered: tracks and showers. Showers are initiated by electromagnetic or hadronic particles produced as the result of the neutrino interaction. They are induced by ${ }^{\complement} \vec{v}_{e}$ charged current (CC) interactions and $\stackrel{c}{v}$ ' neutral current (NC) interactions of all flavours, as well as $\stackrel{c}{\vec{v}}{ }_{\tau} \mathrm{CC}$ interactions with non-muonic tau decays. Muons with a velocity above the Cherenkov threshold produce track-like signatures in the detector. Therefore, track-like events are induced by ${ }^{c} \vec{v}_{\mu}$ CC interactions, as well as ${ }^{c} \vec{v}_{\tau}$ CC interactions with muonic tau decays. In these events the muon is accompanied by a hadronic shower.

In a detailed investigation the potential of the multi-megaton underwater KM3NeT/ORCA detector (Oscillation Research with Cosmics in the Abyss) to perform the NMH measurement has been evaluated [3]. Due to the attainable experimental resolutions for the neutrino energy and zenith angle, the shower channel can provide a substantial contribution to the NMH sensitivity. Therefore, a highly efficient reconstruction of shower-like events with good energy and direction resolution is an essential ingredient for this measurement. The shower reconstruction performance is part of the full detector response matrices used in the ORCA sensitivity calculation, presented in [4]. Further details on the ORCA experiment are given in [3] and the reconstruction of track-like events in ORCA is presented in [5].

\section{Detector}

ORCA is part of KM3NeT phase 2.0 together with the ARCA experiment [6]. Both detectors use the same technology. Key elements of the detectors are digital optical modules (DOMs), equipped with photomultipliers (PMTs), suspended from string-like structures held vertically by a buoy and anchored to the seabed. Each DOM contains 31 3-inch PMTs, 19 in the lower and 12 in the upper hemisphere [7]. The deep sea infrastructure for the ORCA detector is located offshore from Toulon, France, about $10 \mathrm{~km}$ East of the existing ANTARES neutrino telescope [8], at a depth of $2475 \mathrm{~m}$.

The detector configuration is still subject to optimisation. For the presented study the detector consists of 115 strings placed in a semi-random pattern in a circular footprint. Each string hosts 
18 DOMs with a $6 \mathrm{~m}$ vertical spacing. The mean horizontal distance between adjacent strings is roughly $20 \mathrm{~m}$. The instrumented volume is about $3.65 \times 10^{6} \mathrm{~m}^{3}$, corresponding to $3.75 \mathrm{Mton}$ of sea water. Further details are given in [3].

\section{Simulations}

Neutrino and antineutrino induced interactions in the energy range from 1 to $100 \mathrm{GeV}$ have been generated with a software package based on the widely used GENIE [9] neutrino event generator. All particles emerging from a neutrino interaction vertex are propagated with the GEANT4 based software package KM3Sim [10] that has been developed by the KM3NeT collaboration. It generates Cherenkov light and simulates the photon detection taking into account the light absorption and scattering in sea water as well as the DOM and PMT characteristics.

Optical background light arising from the decay of radioactive ${ }^{40} \mathrm{~K}$ in sea water has been simulated by adding an uncorrelated noise rate of $10 \mathrm{kHz}$ per PMT and time-correlated noise on each DOM $(500 \mathrm{~Hz}$ twofold, $50 \mathrm{~Hz}$ threefold, $5 \mathrm{~Hz}$ fourfold and $0.5 \mathrm{~Hz}$ fivefold).

Events have been triggered based on local coincidences (two or more hit PMTs on the same DOM within a small time window). The trigger conditions are chosen such that the overall trigger rate is dominated by atmospheric muon events and not by pure ${ }^{40} \mathrm{~K}$ optical background.

\section{Phenomenology of shower-like events}

In first approximation, a shower event appears as a point-like burst of light in the detector. An electromagnetic shower induced by a single electron appears in the detector as a single Cherenkov ring in a projection onto a plane perpendicular to the shower axis. Similarly, each hadronic shower particle with sufficient energy will be visible as a Cherenkov ring. Therefore, hadronic showers show a large variety of different signatures due to the various possible combinations of initial hadron types, their momenta and the diversity of their hadronic interactions in shower evolution.

A simulated $v_{e} \mathrm{CC}$ example event with $E_{v} \approx 10 \mathrm{GeV}$ and $y \approx 0.5$ is shown in Fig. 1 . The inelasticity of the reaction - also often referred to as Bjorken- $y$ - is given by: $y=\left(E_{v}-E_{e}\right) / E_{v}$. The Cherenkov ring from the electron is clearly visible together with fainter rings from hadronic shower particles.

While electromagnetic showers show only negligible fluctuations in the number of emitted Cherenkov photons and in the angular light distribution, hadronic showers show significant intrinsic fluctuations in the relevant energy range. These intrinsic fluctuations of hadronic showers and the resulting limitations for the energy and angular resolutions have been studied in detail, see [11].

The average light yield is larger for electromagnetic than for hadronic showers. The angular light distribution in the ORCA detector is shown in Fig. 2 for $E_{e}=E_{\text {had }}=5 \mathrm{GeV}$. For both shower types the probability to detect at least one photon within one DOM is maximal at the Cherenkov angle, but the distribution is broader for hadronic than for electromagnetic showers.

Due to the large scattering length in water the different light emission characteristics are conserved over sufficiently large distances, so that information from a large detector volume can contribute to the discrimination between both shower types. In ${ }^{~} \vec{v}_{e}$ CC events, in which both an electromagnetic and a hadronic shower are present and partly overlapping, the angular separation $\phi_{e, \text { had }}$ of 

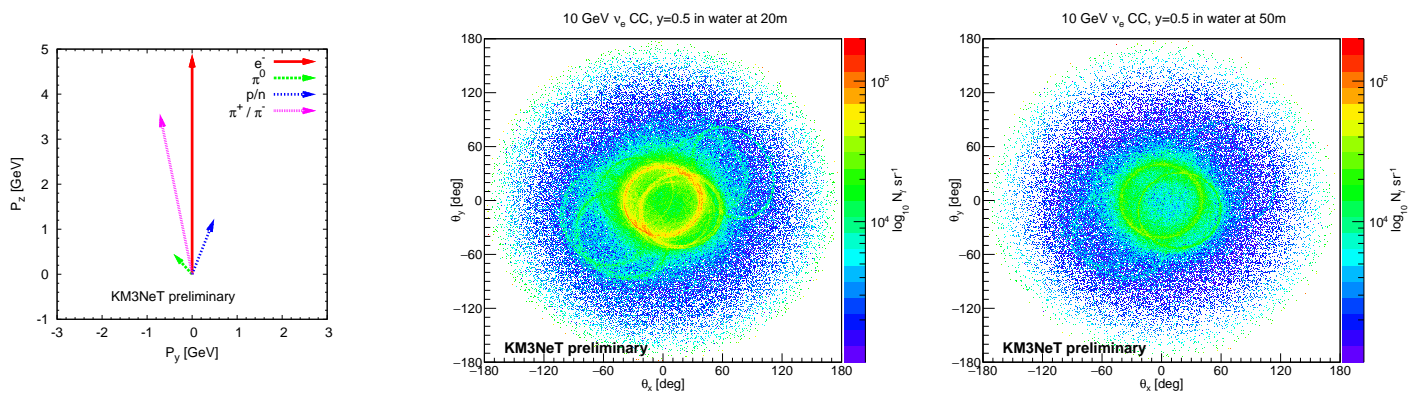

Figure 1: A simulated $v_{e} \mathrm{CC}$ event with $E_{v} \approx 10 \mathrm{GeV}$ and $y \approx 0.5$, rotated in such a way that the electron is in z-direction. Left: illustration of the particles produced in the event. Each arrow represents one particle. The arrow direction and length correspond to the particle momentum in the $p_{y}$ - $p_{z}$-plane. Middle and right: photon distributions in sea water recorded on shells at $20 \mathrm{~m}$ and $50 \mathrm{~m}$ around the neutrino interaction vertex. The Cherenkov ring from the electron is centered around $(0,0)$ with an opening angle of $42^{\circ}$.
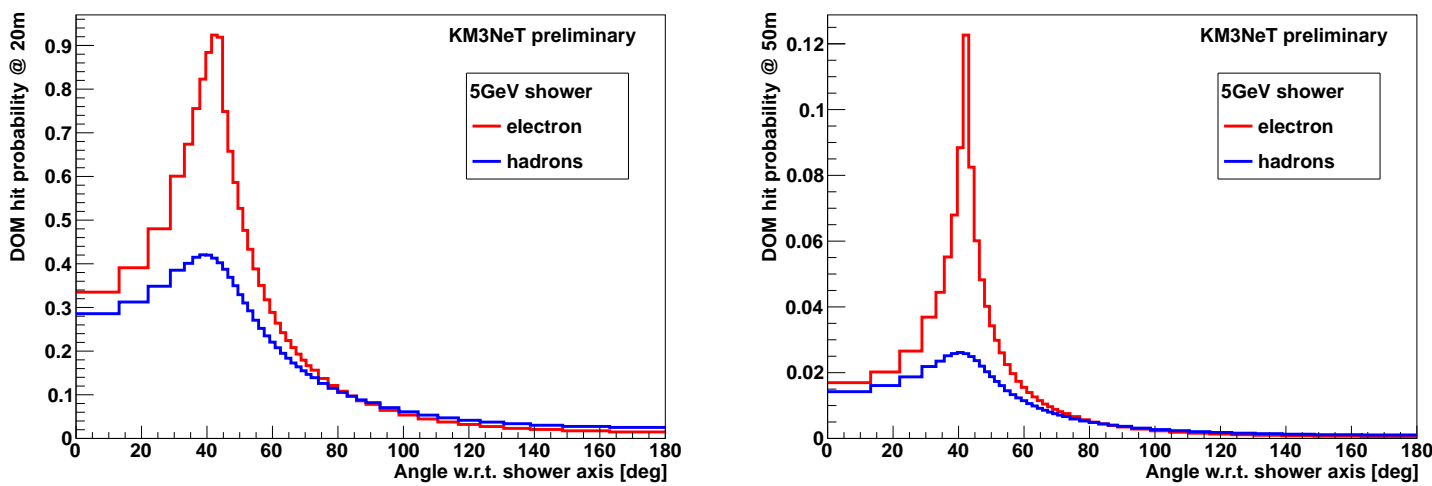

Figure 2: DOM-hit probability (probability to detect at least one photon in an entire multi-PMT optical module) at a distance of $20 \mathrm{~m}$ (left) and $50 \mathrm{~m}$ (right) for showers with $E_{e}=E_{\mathrm{had}}=5 \mathrm{GeV}$ as a function of the angle between shower direction and the vector from shower position to the OM centre.

both showers can help to distinguish between them ${ }^{1}$. This can make an estimation of the inelasticity in $\stackrel{c}{{ }^{\prime}}{ }_{e} \mathrm{CC}$ events feasible.

\section{Shower reconstruction methodology}

A neutrino induced shower-like event is characterised by 8 free parameters: vertex position $\vec{x}_{\mathrm{vtx}}$ and time $t_{\mathrm{vtx}}$, energy $E$, direction $\hat{e}_{s}$ and inelasticity $y$.

The shower reconstruction is performed in two steps. In the first step the vertex is reconstructed based on the recorded time of the PMT signals, commonly called hits, and in the second step the direction, energy and inelasticity are reconstructed based on the number of hits and their distribution in the detector. In both steps a maximum likelihood fit is performed for many different starting shower hypotheses and the solution with the best likelihood is chosen.

This factorization of the fitting procedure works well due to the homogeneity of water and its

\footnotetext{
${ }^{1}$ For neutrino interactions with $E_{v}=10 \mathrm{GeV}$ and $y=0.5$ the mean angle $\phi_{\ell \text {, had }}$ between the outgoing lepton and the outgoing hadronic shower is roughly $25^{\circ}$.
} 
large scattering length which allows for a precise vertex reconstruction independent of the shower direction.

\section{Reconstruction of energy, direction and inelasticity}

In principle, the shower parameters $E, \hat{e}_{s}$ and $y$ can be inferred from the angular light distribution (cf. Fig. 2): the shape is sensitive to the shower type (electromagnetic or hadronic), the integral is approximately proportional to the energy (as the light yield is in first order proportional to the shower energy) and the direction under which this angular light emission profile is present gives the shower direction.

In most $\stackrel{c}{v_{e}} \mathrm{CC}$ events the electron is the dominant particle and produces the brightest Cherenkov ring. Therefore, the reconstruction is designed to find the electron direction and not the neutrino direction.

The shower parameters are reconstructed using a maximum likelihood fit based on the probability that the hit pattern is created by a trial shower hypothesis. A hit selection with a rather high purity of hits from unscattered photons generated by the neutrino event is achieved by selecting hits which are close to the reconstructed vertex, have a small time residual and where the hit PMT is orientated towards the vertex.

For simplification ${ }^{2}$, all selected PMT-hits on the same OM are merged, so that the event is quantified by the number $N_{\text {hits }}^{\mathrm{OM}}$ of hit PMTs for each OM. Ignoring shower-to-shower fluctuations, the probability $P\left(N_{\text {hits }}^{\mathrm{OM}}\right)$ to detect $N_{\text {hits }}^{\mathrm{OM}}$ on a given OM depends on: $E, y$, angle $\theta$ between shower direction $\hat{e}_{s}$ and the vector $\vec{d}$ from the vertex to the OM, distance $d=|\vec{d}|$, and the OM orientation.

To define the probability $P\left(N_{\text {hits }}^{\mathrm{OM}}\right)$ two auxiliary quantities are introduced: the number of expected photons $\left\langle N_{\gamma}\right\rangle$ and its variance $\operatorname{var}\left(\left\langle N_{\gamma}\right\rangle\right)$. The latter is introduced to take the fluctuations in the hadronic shower into account. Both $\left\langle N_{\gamma}\right\rangle$ and $\operatorname{var}\left(\left\langle N_{\gamma}\right\rangle\right)$ depend on $(E, y, \theta, d)$ and are obtained from MC simulations of ${ }^{\stackrel{c}{ } \vec{v}_{e}}$ CC events.

An example distribution of the expected number of photons $\left\langle N_{\gamma}\right\rangle$ as a function of the angle $\theta$ for different inelasticity $y$ intervals is shown in Fig. 3. As the angle $\theta$ is defined with respect to the electron direction, a clear Cherenkov peak of the electron at $42^{\circ}$ is visible. With higher inelasticity this peak becomes fainter due to less energetic electrons, while the number of expected photons in the 'off-peak region' increases due to the more energetic hadronic showers. Therefore, these PDF tables provide sensitivity to the inelasticity from the ratio of the peak to off-peak regions.

\section{Shower reconstruction performance}

The performance of the shower reconstruction is studied on upgoing, i.e. with zenith angle larger $90^{\circ}$, neutrino events simulated as described in Sec. 3. Events have been selected according to reconstruction quality and containment criteria. For all plots the conventional atmospheric neutrino flux following the Bartol model [12] is assumed.

Note, that the reconstruction performance is also essential for the event classification into track- and shower-like events, which is in general taken into account in the NMH sensitivity calculation, c.f. [3, 4]. This classification has not been applied here.

\footnotetext{
${ }^{2}$ This simplification is justified by the fact that an $\mathrm{OM}$ in principle measures the intensity of the shower event at a given spacial position. Of course, the multi-PMT structure is needed to estimate the shower intensity from $N_{\text {hits }}^{\mathrm{OM}}$.
} 


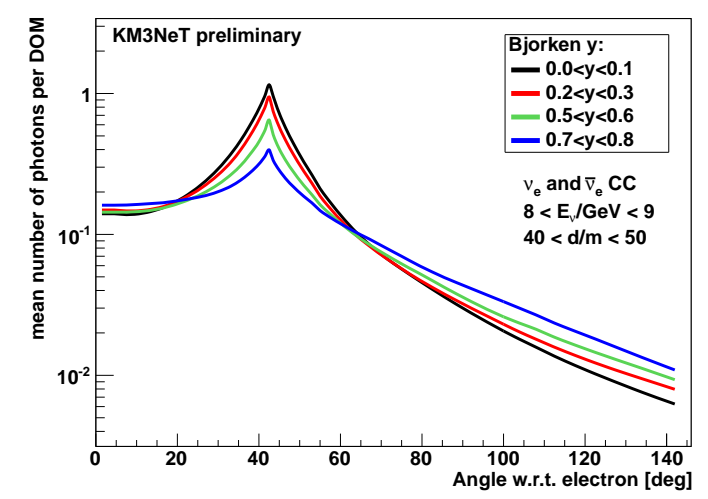

Figure 3: Number of expected photons $\left\langle N_{\gamma}\right\rangle$ as a function of angle $\theta$ between the shower direction, which is the electron direction, and the vector from the vertex to the $\mathrm{OM}$ for $v_{e}$ and $\bar{v}_{e} \mathrm{CC}$ events in different inelasticity $y$ intervals, and for neutrino energies of $8<E / \mathrm{GeV}<9$ and at distances of $40<d / \mathrm{m}<50$.

\section{Effective volume}

The effective volume for upgoing shower-like neutrino interaction channels is shown in Fig. 4 (left) as a function of neutrino energy. In saturation $\left(3.6 \mathrm{Mm}^{3}\right)$ the instrumented volume is nearly reached. The turn-on is slightly steeper for $\bar{v}_{e} \mathrm{CC}$ than for $v_{e} \mathrm{CC}$ events due to the lower average inelasticity. Events with a lower inelasticity $y$ (higher fraction of electromagnetic shower component) have a higher probability to be well reconstructed. Due to the invisible outgoing neutrino(s)

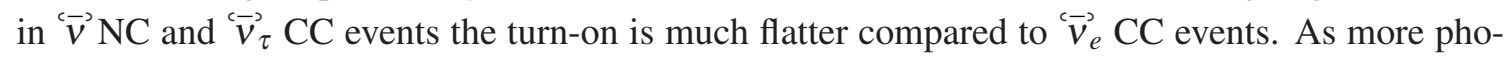
tocathode area is looking down- than upwards, the turn-on is on average about $25 \%$ steeper for vertical upgoing (more important for NMH determination) than for horizontal events.


Figure 4: Left: effective volumes in $\mathrm{Mm}^{3}\left(10^{6} \mathrm{~m}^{3}\right)$ for different upgoing shower-like neutrino interaction

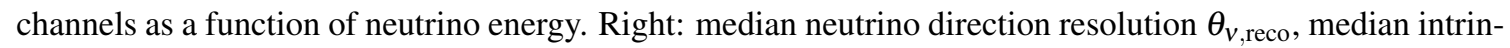
sic scattering angle $\theta_{v, \mathrm{e}}$ and median electron direction resolution $\theta_{\mathrm{e}, \text { reco }}$ as a function of neutrino energy for upgoing $v_{e} \mathrm{CC}$ and $\bar{v}_{e} \mathrm{CC}$ events, separately.

\section{Vertex resolution}

The spatial resolution of the shower vertex is roughly Gaussian with $\sigma \approx 0.5-1 \mathrm{~m}\left(E_{v}\right.$ and $y$ dependent) and is dominated by the error on the longitudinal component in neutrino direction. 


\section{Direction resolution}

The median neutrino direction resolution (angle $\theta_{v \text {,reco }}$ between reconstructed direction and neutrino direction) is better than $10^{\circ}$ for energies above $8 \mathrm{GeV}(5 \mathrm{GeV})$ for $v_{e}\left(\bar{v}_{e}\right) \mathrm{CC}$ events and is shown as a function of neutrino energy in Fig. 4 (right). In addition, the median intrinsic scattering angles $\theta_{v, \mathrm{e}}$ and the median resolution with respect to the electron direction $\theta_{\mathrm{e}, \text { reco }}$ are shown. For the relevant energy range the median electron direction resolution is smaller than the intrinsic scattering angle and the median neutrino direction resolution, verifying that the reconstruction actually has the ability to find the electron in $\stackrel{\mathrm{v}}{e}_{e} \mathrm{CC}$ events.

\section{Inelasticity resolution}

The resolution on the inelasticity $y$ for a low, medium and high $y$ range is shown in Fig. 5 (left) for ${ }^{c} \vec{v}_{e}$ CC events with $8 \mathrm{GeV}<\mathrm{E}_{v}<12 \mathrm{GeV}$. Due to the $y$ sensitivity the $y_{\text {reco }}$ distribution is different for $v_{e}$ and $\bar{v}_{e}$ CC events leading to some separation power between both channels and also

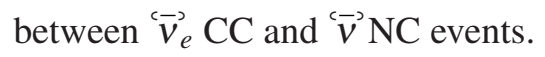
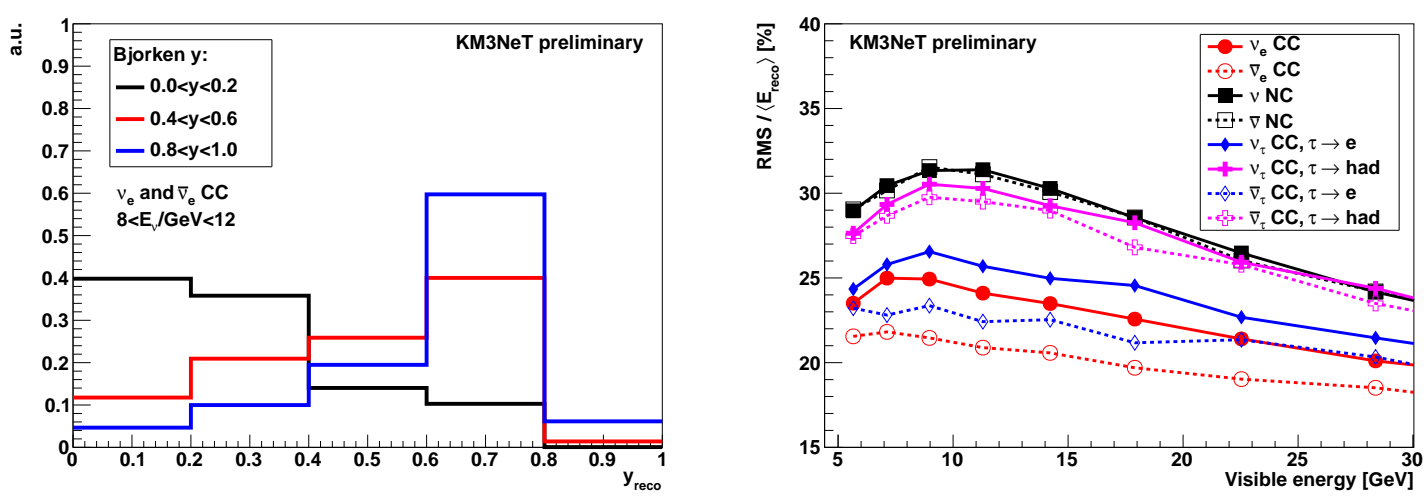

Figure 5: Left: distribution of reconstructed inelasticity $y_{\text {reco }}$ for three different true $y$ ranges $(0<y<0.2$, $0.4<y<0.6$ and $0.8<y<1$ ) for $v_{e} \mathrm{CC}$ and $\bar{v}_{e} \mathrm{CC}$ events with $8 \mathrm{GeV}<\mathrm{E}_{v}<12 \mathrm{GeV}$. Right: relative energy resolution $\mathrm{RMS} /\left\langle E_{\text {reco }}\right\rangle$ as a function of the visible energy $E_{\mathrm{vis}}$ for shower-like neutrino interaction channels.

\section{Energy resolution}

The reconstructed energy is systematically higher than the neutrino energy. Therefore, a linear energy correction depending on the reconstructed zenith angle $\theta_{\text {reco }}$, inelasticity $y_{\text {reco }}$ and energy $E_{\text {reco }}$ is applied. The 3-dimensional correction function has been calculated from MC such that the median reconstructed energy is equal to the neutrino energy in ${ }^{c} \vec{v}_{e} \mathrm{CC}$ events.

After this energy correction, the energy resolution is very well described by a Gaussian. The relative energy resolution - given as RMS over mean reconstructed energy $\left\langle E_{\text {reco }}\right\rangle$ - is better than $25 \%(22 \%)$ for neutrino energies above $7 \mathrm{GeV}$ for upgoing $v_{e}\left(\bar{v}_{e}\right) \mathrm{CC}$ events and is shown as a function of visible energy $E_{\mathrm{vis}}$ in Fig. 5 (right) together with the resolution for the other shower-like neutrino interaction channels. $E_{\mathrm{vis}}$ is defined as the difference between the energy of the incoming neutrino and the outgoing neutrino(s) from the primary neutrino interaction (NC events) or $\tau$-decay $\left(\vec{v}_{\tau}\right.$ CC events). 
Due to the smaller light yield of hadronic showers compared to electromagnetic showers, the ration $\left\langle E_{\text {reco }}\right\rangle / E_{\text {vis }}$ is different for each neutrino interaction channel and energy dependent. This leads also to different turn-on behaviours in the effective volume for both shower types, and consequently to different compositions (in terms of electromagnetic and hadronic shower components) of well reconstructed neutrino events. The latter explains the energy resolution behaviour below $E_{\mathrm{vis}} \lesssim 10 \mathrm{GeV}$. The resolution is worse for events with higher average contribution from hadronic showers which show large fluctuations [11].

\section{Conclusions}

Detailed simulations show that the large scattering length in water allows to achieve good direction and energy resolutions for shower-like events in the ORCA detector, while the effective volume in saturation is close to the instrumented volume. Additionally, it is possible to distinguish on a statistical basis the signatures of multi-GeV electromagnetic and hadronic showers. Therefore, the shower reconstruction can find the electron direction in ${ }^{c} \vec{v}_{e}$ CC events and is able to gain access to the interaction inelasticity $y$. The directional resolution is dominated by the intrinsic scattering angle between the neutrino and the outgoing electron. The $y$ sensitivity allows to compensate for different light yields in electromagnetic and hadronic showers and improves the energy resolution achieving a relative resolution better than $25 \%$ (22\%) for upgoing $v_{e}\left(\bar{v}_{e}\right)$ CC events. A separation of $v_{e}$ and $\bar{v}_{e} \mathrm{CC}$ and of ${ }^{\complement} \vec{v} \mathrm{NC}$ events may also be possible on a statistical basis. The resulting gain in $\mathrm{NMH}$ sensitivity will be investigated in further studies.

Investigations of detector configurations with larger vertical spacings and consequently larger instrumented volumes indicate that for shower-like neutrino events the reported energy and direction resolutions show a minor degradation, while the corresponding effective volumes saturate at higher values, however, with less steep turn-on. The possible gain in NMH sensitivity due to a detector configuration optimisation will be investigated in further studies.

\section{References}

[1] E. Akhmedov, S. Razzaque, A. Smirnov, JHEP 1302 (2013) 082.

[2] M. Ribordy and A.Yu. Smirnov, Phys.Rev. D 87 (2013) 113007.

[3] J. Brunner, for the KM3NeT Coll., PoS(ICRC2015)1140.

[4] M. Jongen, for the KM3NeT Coll., PoS(ICRC2015)1092.

[5] S. Galata, for the KM3NeT Coll., PoS(ICRC2015)1102.

[6] P. Piattelli, for the KM3NeT Coll., PoS(ICRC2015)1158.

[7] R. Bruijn, for the KM3NeT Coll., PoS(ICRC2015)1157.

[8] M. Ageron et al., Nucl. Instrum. Meth. A 656 (2011) 11.

[9] C. Andreopoulos et al., Nucl. Instrum. Meth. A 614 (2010) 87.

[10] A.G. Tsirigotis et al., PoS(Neutel 2013)080.

[11] J. Hofestädt, for the KM3NeT Coll., PoS(ICRC2015)1084.

[12] G.D. Barr et al., Phys.Rev. D 70 (2004) 023006. 